\title{
DEVELOPMENT OF LEVEL 1B CALIBRATION AND VALIDATION READINESS, IMPLEMENTATION AND MANAGEMENT PLANS FOR GOES-R
}

\author{
David B. Kunkee, Robert W. Farley, Betty P. Kwan, James H. Hecht, Richard L. Walterscheid, \\ Seth G. Claudepierre, Rebecca L. Bishop, Lynette J. Gelinas, Tamitha M. Skov, and Frank J. De Luccia
}

\author{
The Aerospace Corporation \\ PO Box 92957 \\ Los Angeles, CA 90009-2957
}

\begin{abstract}
A complement of Readiness, Implementation and Management Plans (RIMPs) to facilitate management of post-launch product test activities for the official Geostationary Operational Environmental Satellite (GOESR) Level 1b (L1b) products have been developed and documented. Separate plans have been created for each of the GOES-R sensors including: the Advanced Baseline Imager (ABI), the Extreme ultraviolet and X-ray Irradiance Sensors (EXIS), Geostationary Lightning Mapper (GLM), GOES-R Magnetometer (MAG), the Space Environment InSitu Suite (SEISS), and the Solar Ultraviolet Imager (SUVI). The GOES-R program has implemented these RIMPs in order to address the full scope of $\mathrm{Cal} / \mathrm{Val}$ activities required for a successful demonstration of GOES-R L1b data product quality throughout the three validation stages: Beta, Provisional and Full Validation. For each product maturity level, the RIMPs include specific performance criteria and required artifacts that provide evidence a given validation stage has been reached, the timing when each stage will be complete, a description of every applicable Post-Launch Product Test (PLPT), roles and responsibilities of personnel, upstream dependencies, and analysis methods and tools to be employed during validation. Instrument level PostLaunch Tests (PLTs) are also referenced and apply primarily to functional check-out of the instruments.
\end{abstract}

Index Terms - Calibration, Validation, Advanced Baseline Imager, GOES

\section{INTRODUCTION}

Development of the L1b RIMPs involved a complex process that considered interactions between several different L1b products many times led by different science leads and organizations. $\mathrm{Cal} / \mathrm{Val}$ activities for each $\mathrm{L} 1 \mathrm{~b}$ product also had to accommodate a fixed GOES-R Cal/Val schedule and performance requirements set. To accomplish this, the $\mathrm{L} 1 \mathrm{~b}$ team worked directly with the science leads from NOAA's
Satellite Applications and Research (STAR), NOAA's National Centers for Environmental Information (NCEI) in Boulder, Colorado and the GOES-R program to determine the scope of the documents, and then to organize, coordinate, and develop all of the L1b RIMPs. During the RIMP development period, hundreds of pre-existing PLPTs were reviewed and revised before finalizing plans for the baseline post-launch $\mathrm{Cal} / \mathrm{Val}$. Although the PLPT format offered a standardized format to describe Validation Events (VE's) during Cal/Val, the complement of PLPTs for each sensor were quite varied in technical content, duration and test output or artifacts.

\section{ADVANCED BASELINE IMAGER (ABI)}

The baseline Cal/Val plan for ABI includes 21 Post-Launch Tests (PLTs) in order to validate initial on-orbit instrument functional performance. After successful execution of the PLTs, the L1b data is assessed at Beta product maturity. Detailed product testing to advance data product maturity is performed during the subsequent Provisional and Full Validation periods, as described in the corresponding RIMP's and PLPT's, and shown graphically in Figure 1.

Development of the RIMPs involved reviewing existing $\mathrm{Cal} / \mathrm{Val}$ documents and assisting with development of the requirements verification cross reference matrix. This mapping helped identify risks associated with validation of required performance during low-light operations and the new requirement for GOES-R to operate during orbit adjust maneuvers. These risks were carefully considered by the GOES-R program for mitigation and closure through additional testing and analysis. A top-level $\mathrm{Cal} / \mathrm{Val}$ schedule for the ABI provisional and full validation activities was also developed to help project managers to categorize, prioritize and schedule tests, analyses, and product tool development. The schedule also helped to identify key performance verification activities and dependencies. A graphical representation of the ABI $\mathrm{Cal} / \mathrm{Val}$ schedule is shown in Figure 2. 
Beta VEs

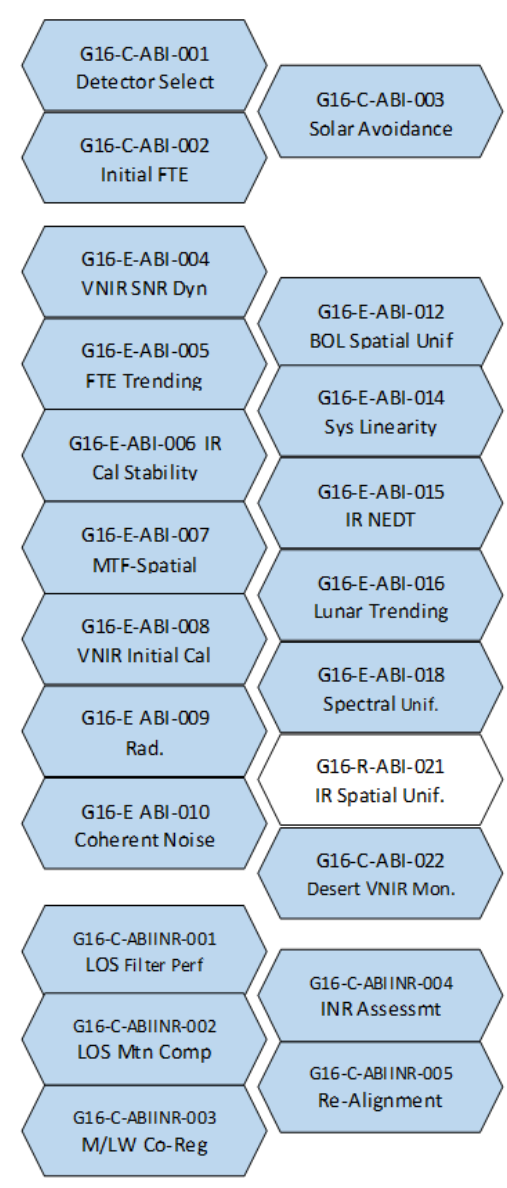

Provisional VEs

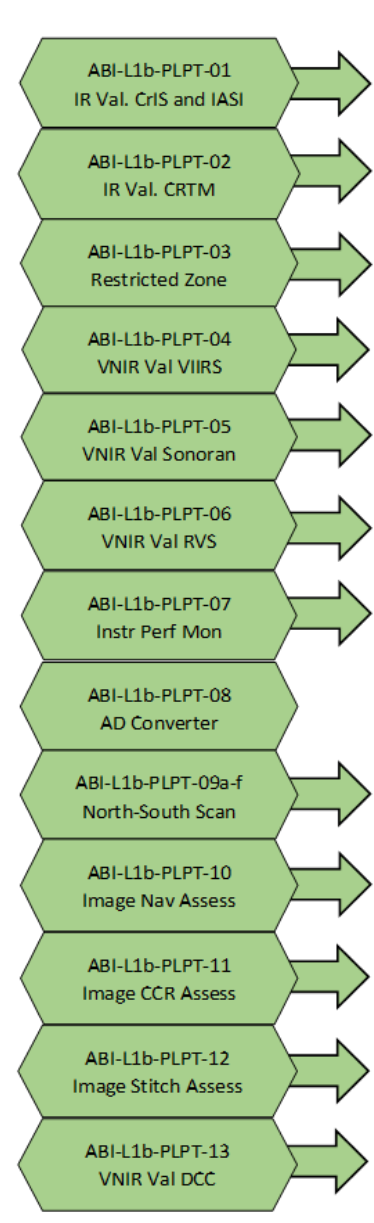

Full Validation

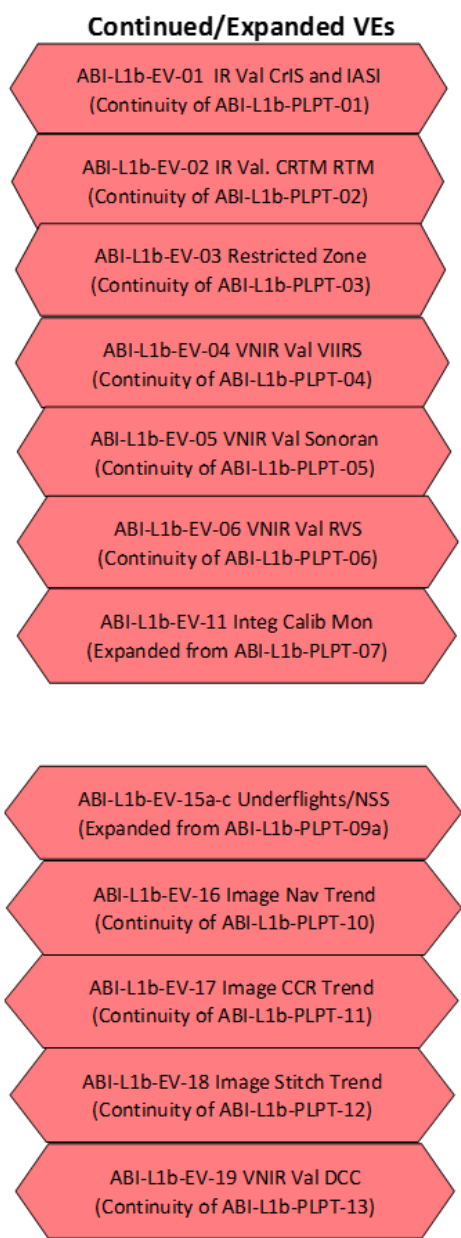

New/Additional VEs

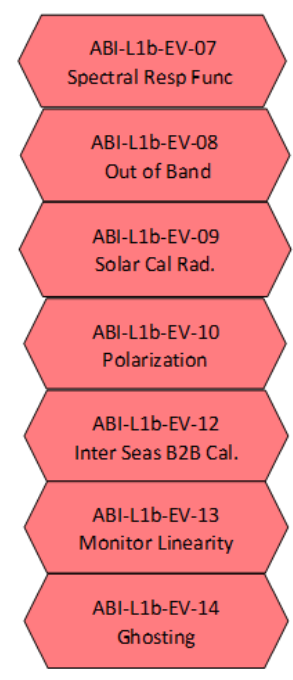

\begin{tabular}{|l|lll|l|}
$\square$ & Reserve & $\begin{array}{l}\text { Within PLT } \\
\text { Scope }\end{array}$ & $\begin{array}{l}\text { Within PLPT } \\
\text { Scope }\end{array}$ & $\begin{array}{l}\text { Extended } \\
\text { Validationn }\end{array}$ \\
\hline
\end{tabular}

Figure 1. Graphical representation of the Advanced Baseline Imager (ABI) Cal/Val Validation Events (VEs). The Post Launch Tests (PLTs) VEs are shown in blue and Post-Launch Product Tests (PLPTs) VEs are shown in Green and Red. Arrows indicate PLPTs that are continued throughout provisional and Full Validation periods.

\section{THE GEOSTATIONARY LIGHTNING MAPPER (GLM)}

The Level $1 \mathrm{~b}$ (L1b) and 2 (L2) RIMPs for the PLTs and PLPTs required from the GOES-R Geostationary Lightning Mapper (GLM) [3] were also developed and organized as part of the overall GOES-R Cal/Val effort. PLT and PLPT events and products include Level 1b GLM Events and Level 2 Groups (strokes) and Flashes. The GLM RIMP focuses on those aspects of PLTs and PLPTs (and their preparation activities) that apply or impact the validation of the GLM product science quality. The GLM RIMP differed from other RIMPs by the overall number of the products identified. There are a total of 44 PLPTs in 13 series and each will go to Full data product maturity. Each series refers to a distinct assessment (e.g., Detection Efficiency and False Event Rate) by a specific means (well characterized medium to long range networks with medium to high detection efficiencies and low false alarm rates). In each member of the series a number of PLPTs were possible (e.g., one for each network). The $\mathrm{Cal} / \mathrm{Val}$ challenge was to develop tools for the multitude of PLPTs, identify sites that provide adequate site diversity, group them appropriately according to specific capabilities, and prepare for the volume of $\mathrm{Cal} / \mathrm{Val}$ data that the various data sources would generate 


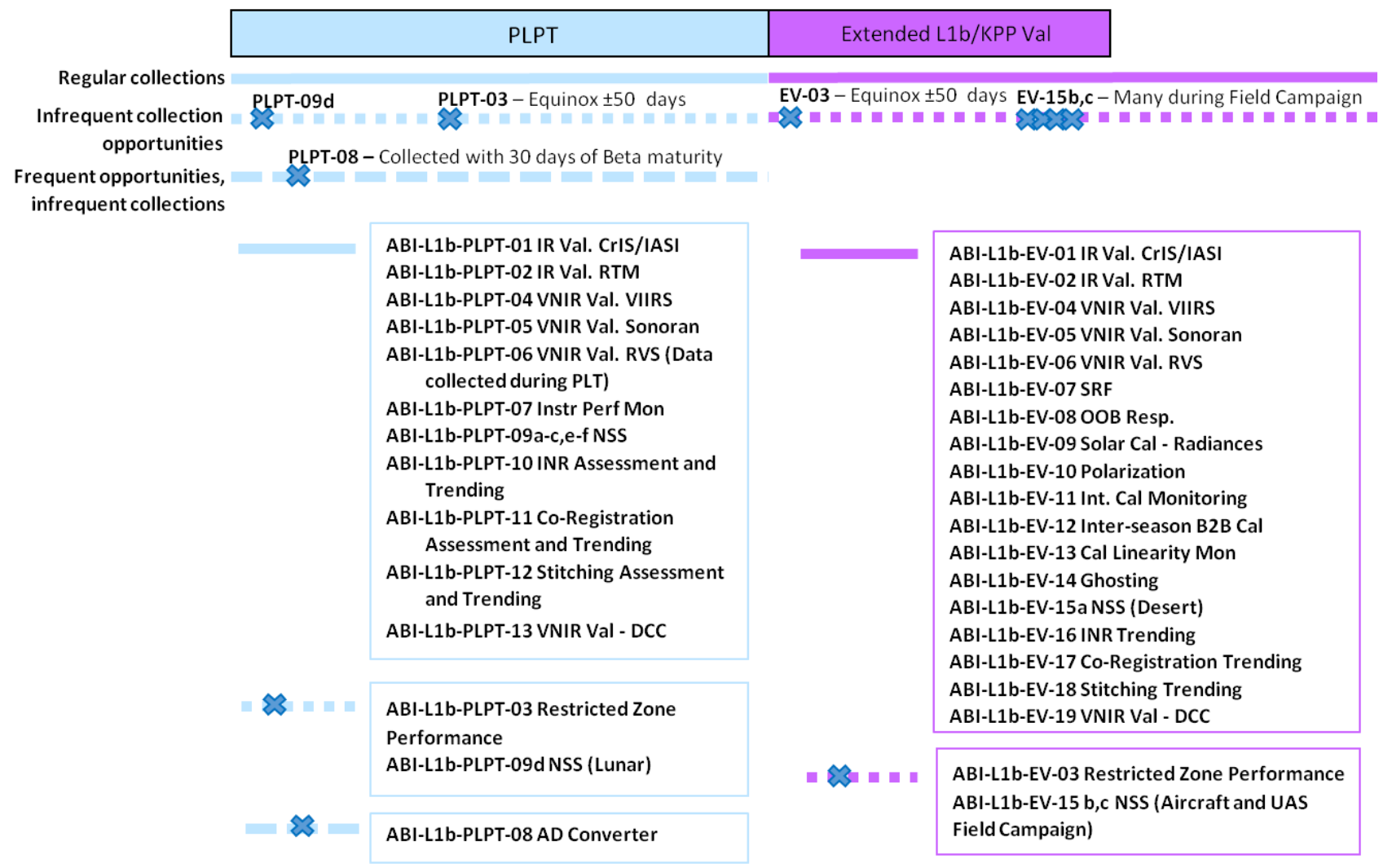

Figure 2. Schedule of Cal/Val activities for ABI Provisional and Full Validation.

(including campaign and satellite data). The reviews and discussions necessary to create the RIMP led to extensive consultations with the $\mathrm{Cal} / \mathrm{Val}$ teams to better organize and improve the GLM Cal/Val mission.

\section{GOES-R SPACE WEATHER INSTRUMENTS AND MAGENTOMETER}

A summary of the GOES-R space weather instrument complement can be found online [4]. We present the toplevel aspects of the RIMP for each of these instruments in this Section.

The Extreme ultraviolet and X-ray Irradiance Sensors (EXIS) includes the X-Ray Sensor (XRS), Extreme UltraViolet Sensor (EUVS), and Solar Pointing Sensor (SPS). The XRS and EUVS have heritage sensors on other GOES satellites, although both have been modified for the GOES-R series. The SPS measures the pointing of the EXIS relative to the center of the Sun. For EXIS, a total of 12 PLTs are necessary to establish Beta maturity and 14 PLPTs required to establish provisional maturity. Of these, 7 PLTs will be transitioned to NCEI, and continued for the lifetime of the instrument, however, to establish full validation for the EXIS data products, NCEI requires results from only 2 of the PLPTs.

The Space Environment In-Situ Suite (SEISS) includes the Magnetospheric Particle Sensor - Low energy (MPS-LO), Magnetospheric Particle Sensor - High energy (MPS-HI), Solar and Galactic Proton Sensor (SGPS) and Energetic Heavy Ion Sensor (EHIS). These sensors provide a more comprehensive view of charged particle radiation at geostationary orbit compared with previous GOES satellites. For SEISS, a total of 8 PLTs are defined to support Beta product maturity including 5 PLTs that will be transitioned to NCEI to be carried out over the lifetime of the instrument for trending and continued characterization. A total of 6 PLPTs have been defined to support provisional maturity and 5 there are five of these required for full data product validation over a period of $\sim 1$ year from the beginning of $\mathrm{Cal} / \mathrm{Val}$.

The Solar Ultraviolet Imager (SUVI) is a normal incidence telescope in the Ritchey-Chrétien configuration with ChargeCoupled Device (CCD) detectors at the Cassegrain focus. Six narrow wavelength bands are utilized to image different 
features of the full-disk Sun. Beta product maturity for SUVI is supported by 9 PLTs and all but one of these tests will be transitioned to NCEI and carried out over the lifetime of the instrument. There are six PLPTs that are utilized to validate the instrument calibration. However, they need to be performed at certain intervals over the instrument lifetime in order to maintain the fidelity of the SUVI data.

Provisional data product validation is achieved upon successful completion of the first two PLPTs that will occur $\sim 6$ months after launch. Full validation occurs upon completion of the last four PLPTS that will occur $\sim 240$ days after launch. The RIMP process allowed the identification of specific criteria, discussed in the $\mathrm{Cal} / \mathrm{Val}$ plan but not previously identified from the PLPTs alone that must be met for full validation.

The GOES-R Magnetometer (MAG) consists of 2 boommounted fluxgate magnetometers capable of providing magnetic field measurements with a temporal resolution 5 times greater $(10 \mathrm{~Hz})$ than that of the heritage GOES-N,O, P system. The inboard magnetometer is located at approximately $6.3 \mathrm{~m}$ from the baseplate of the boom, and the outboard magnetometer is located at approximately $8.5 \mathrm{~m}$ from the baseplate of the boom. For the MAG Cal/Val, 4 PLTs have been defined to support Beta product maturity. Beyond Beta maturity, 5 PLPTs have been defined to support MAG, 2 of which are required for provisional maturity and 4 of the 5 PLPTs are required for full validation. These PLPTs include, for example, comparisons with well-established, empirical magnetic field models of the near-Earth space environment (e.g., Olson-Pfitzer [5], and Tsyganenko [6]).

\section{SUMMARY AND STATUS}

The GOES-R (now GOES-16) launched on 19 November 2016 from the Kennedy Space Center, FL USA and reached
Geosynchronous Orbit on November 29. Post Launch testing is planned to commence in accordance with the RIMPs developed under the above described effort with public release of the first ABI L1b images scheduled in January 2017. Data products and images from other GOES$\mathrm{R}$ sensors are also scheduled. Accordingly, the earliest data to be made public is planned from the GOES-R MAG in December 2016 and the latest sensor L1b imagery scheduled for public release will be from GLM in April 2017.

This presentation will discuss the formulation of the GOES$\mathrm{R}$ instrument RIMPs highlighting the application of system engineering practices. Examples include mapping program requirements to justify each PLPT, identification of requirements to successfully execute each of the identified PLPTs such as software tools and data access, schedule constraints, and data product interdependencies.

\section{REFERENCES}

[1] http://www.goes-r.gov/spacesegment/abi.html

[2] http://www.goes-r.gov/education/ABI-bands-quickinfo.html

[3] http://www.goes-r.gov/spacesegment/glm.html

[4] http://www.goes-r.gov/education/docs/Spaceweather.pdf

[5] Olson, W. P., and K. A. Pfitzer, Magnetospheric magnetic field modeling, Tech. Rep. Annual Report, McDonnell-Douglas Astronautics Co., Huntington Beach, CA.

[6] Tsyganenko, N. A. (1989), A magnetospheric magnetic field model with a warped tail current sheet, Planet. Space Sci., 37, 5-20, DOI:10.1016/0032-0633(89)90066-4. 